\title{
Static PDEs for time-dependent control problems
}

\author{
A. VLADIMIRSKY ${ }^{\dagger}$ \\ Department of Mathematics, Cornell University, Ithaca, NY 14853, USA
}

[Received 11 June 2004 and in revised form 1 March 2006]

\begin{abstract}
We consider two different non-autonomous anisotropic time-optimal control problems.
For the min-time-from-boundary problem, we show that the value function is recovered as a viscosity solution of a static Hamilton-Jacobi-Bellman partial differential equation $H(\nabla u(\boldsymbol{x}), u(\boldsymbol{x}), \boldsymbol{x})=1$. We demonstrate that the space-marching Ordered Upwind Methods (introduced in [29] for the autonomous control) can be extended to this non-autonomous case. We illustrate this approach with several numerical experiments.

For the min-time-to-boundary problem, where no reduction to a static PDE is possible, we show how the space-marching methods can be efficiently used to approximate individual level sets of the time-dependent value function.
\end{abstract}

\section{Introduction}

Min-time autonomous control problems are among the most studied in the control literature (see, for example, [1], [2], [12], and references therein.) Time-dependent (non-autonomous) control problems can be easily reduced to the above case by adding the time as an extra dimension to the system-state; however, such a reduction can be expensive from the practical point of view since it also increases the dimensionality of the computational domain. The goal of this paper is to treat nonautonomous control problems directly - by working in the original state-space whenever possible. In discussing the properties of the corresponding value functions we provide detailed proofs only when there is a significant difference from the standard arguments employed in the autonomouscontrol setting.

We consider two different anisotropic non-autonomous time-optimal control problems on an open bounded domain ${ }^{1} \Omega \subset \mathbb{R}^{2}$.

In the first problem, the value function $u$ is the minimum time for driving the vehicle to $x \in \Omega$ starting from any point $\boldsymbol{y}_{0} \in \partial \Omega$, and enduring the entry time-penalty $q\left(\boldsymbol{y}_{0}\right)$. Normally, a value function for a non-autonomous control problem is also time-dependent. In this case, we demonstrate that, despite the time-dependence of the vehicle's dynamics, a time-independent value function $u(\boldsymbol{x})$ can be defined, and that $u(\boldsymbol{x})$ can be recovered as the unique viscosity solution for a static Hamilton-Jacobi-Bellman PDE of the form

$$
\begin{array}{ll}
H(\nabla u, u, \boldsymbol{x})=\|\nabla u(\boldsymbol{x})\| F\left(\boldsymbol{x}, \frac{\nabla u(\boldsymbol{x})}{\|\nabla u(\boldsymbol{x})\|}, u(\boldsymbol{x})\right)=1, & \boldsymbol{x} \in \Omega, \\
u(\boldsymbol{x})=q(\boldsymbol{x}), & \boldsymbol{x} \in \partial \Omega .
\end{array}
$$

This interpretation serves as a basis for the computational methods for hybrid control in [31] and is also at the core of the recent work by Sethian and Shan on non-autonomous isotropic control [28].

\footnotetext{
${ }^{\dagger}$ E-mail: vlad@math.cornell.edu

1 For the sake of notational clarity we restrict our discussion to $\mathbb{R}^{2}$; all results can be restated for $\mathbb{R}^{n}$ and on manifolds.
} 
However, to the best of our knowledge, the theoretical aspects of this re-casting have not appeared elsewhere and thus we are covering them in detail in Section 2

We show that additional controllability assumptions yield an upper bound for the angle between the characteristics of the PDE (1) and the gradient lines of its viscosity solution. In a joint work with Sethian [29, 30], this observation was used to design non-iterative Ordered Upwind Methods (OUMs) for the autonomous control problems, later extended to the non-autonomous and hybrid control in [31]. In Section 3 we briefly describe these methods, and derive sharper (localized) upper bounds for the characteristic-gradient angles to improve the computational efficiency. The approach is illustrated with several min-time-from-boundary examples in 3.1 .

In the second problem, the value function $v$ is the minimum time needed for a vehicle to leave the domain if it starts at $\boldsymbol{x} \in \Omega$ and endures an exit time-penalty $\hat{q}$ on the boundary. For this mintime-to-boundary problem, the time-dependent dynamics corresponds to a time-dependent value function $v(\boldsymbol{x}, t)$ even if the exit time-penalty $\hat{q}$ is stationary. (The optimal path for reaching $\partial \Omega$ from $\boldsymbol{x}$ truly depends on the starting time $t$.) Under a standard set of assumptions, $v(\boldsymbol{x}, t)$ can be obtained as the unique viscosity solution for a Hamilton-Jacobi-Bellman PDE

$$
\begin{array}{ll}
v_{t}(\boldsymbol{x}, t)-H\left(-\nabla_{x} v(\boldsymbol{x}, t), t, \boldsymbol{x}\right)=0, & \boldsymbol{x} \in \Omega, t \in \mathbb{R}, \\
v(\boldsymbol{x}, t)=\hat{q}(\boldsymbol{x}, t)+t, & \boldsymbol{x} \in \partial \Omega, t \in \mathbb{R} .
\end{array}
$$

This interpretation is fairly standard and we discuss it only briefly in Section 4 . We then proceed to demonstrate that, if $(\hat{q}(\boldsymbol{x}, t)+t)$ is a monotone function of time, then any individual level set of $v(\boldsymbol{x}, t)$ can be computed separately by converting to a dual (min-time-from-boundary) problem and solving the latter using OUMs.

We note that efficient methods for static Hamilton-Jacobi PDEs have recently become an active research area. Alternatives to OUMs include Dijkstra-like methods based on cell-mapping techniques [17] or bisimulations [5, 6], time-marching methods based on paraxial approximations [25, 26], and alternating-direction Gauss-Seidel relaxations [9, 4], which also served as the basis for the recent family of "fast sweeping methods" [34, 36, 18]. To the best of our knowledge, these techniques have been used only for Hamiltonians depending directly on $\nabla u$ but not on $u$ itself. However, we believe that it should also be possible to extend them to the problems studied here, and we note that the control-theoretic analysis in Sections 2 and 4 is largely independent of our numerical approach.

We conclude by discussing the limitations of our method and possible future extensions in Section 5

\section{Time-dependent case: min-time from the boundary}

Given an open bounded domain $\Omega \subset \mathbb{R}^{2}$, the goal is to find the minimum time necessary to reach a point $x \in \Omega$ starting from any point on $\partial \Omega$. The vehicle's dynamics in $\Omega$ is defined by

$$
\begin{aligned}
\boldsymbol{y}^{\prime}(t) & =f(\boldsymbol{y}(t), \boldsymbol{a}(t), t) \boldsymbol{a}(t), \\
\boldsymbol{y}\left(\tau=q\left(\boldsymbol{y}_{0}\right)\right) & =\boldsymbol{y}_{0} \in \partial \Omega,
\end{aligned}
$$

where $q: \partial \Omega \rightarrow \mathbb{R}_{+, 0}$ is an entry time-penalty, $\boldsymbol{y}(t)$ is the vehicle's position at the time $t \in$ $[\tau,+\infty), f: \bar{\Omega} \times S_{1} \times \mathbb{R}_{+, 0} \rightarrow \mathbb{R}_{+, 0}$ is the speed of motion in the chosen direction $\boldsymbol{a}(t)$, and $\mathcal{A}=\left\{\right.$ measurable $\left.\boldsymbol{a}: \mathbb{R}_{+, 0} \rightarrow S_{1}\right\}$ is the set of admissible controls. Unless otherwise explicitly 
specified, we will assume that both $f$ and $q$ are Lipschitz-continuous and that there exist constants $F_{1}, F_{2}, q_{1}, q_{2}$ and continuous functions $f_{1}, f_{2}: \bar{\Omega} \rightarrow \mathbb{R}_{+}$such that

$$
\begin{array}{cc}
0<F_{1} \leqslant f_{1}(\boldsymbol{y}) \leqslant f(\boldsymbol{y}, \boldsymbol{a}, t) \leqslant f_{2}(\boldsymbol{y}) \leqslant F_{2}<\infty & \forall \boldsymbol{y} \in \bar{\Omega}, \forall \boldsymbol{a} \in S_{1}, \forall t \in \mathbb{R}_{+, 0} ; \\
0 \leqslant q_{1} \leqslant q(\boldsymbol{y}) \leqslant q_{2}<\infty & \forall \boldsymbol{y} \in \partial \Omega .
\end{array}
$$

For notational convenience, we will also define the anisotropy coefficient $\Upsilon=F_{2} / F_{1}$. Strictly speaking, since $F_{1}$ and $F_{2}$ are global bounds, the coefficient $\Upsilon$ reflects the measure of anisotropy only in the homogeneous domain (i.e., when $f(\boldsymbol{x}, \boldsymbol{a}, t)=f(\boldsymbol{a}, t)$ ). In what follows, we use $\Upsilon$ to derive the worst-case-scenario computational complexity of the algorithms. In local estimates, we will further use two more relevant constants: a neighborhood anisotropy coefficient

$$
\Upsilon_{d}(\boldsymbol{x})=\max _{\boldsymbol{y}_{1}, \boldsymbol{y}_{2} \in B_{d}(\boldsymbol{x}) \cap \bar{\Omega}} \frac{f_{2}\left(\boldsymbol{y}_{1}\right)}{f_{1}\left(\boldsymbol{y}_{2}\right)},
$$

where $B_{d}(\boldsymbol{x})$ is a ball of radius $d$ centered at $\boldsymbol{x}$, and a local anisotropy coefficient

$$
\Upsilon(\boldsymbol{x})=\frac{f_{2}(\boldsymbol{x})}{f_{1}(\boldsymbol{x})}=\lim _{d \rightarrow 0} \Upsilon_{d}(\boldsymbol{x}) .
$$

We now define the total time/cost for every trajectory

$$
\mathcal{J}\left(\boldsymbol{x}, \boldsymbol{a}(\cdot), \boldsymbol{y}_{0}, \tau\right)= \begin{cases}\min \{t \in[\tau,+\infty) \mid \boldsymbol{y}(t)=\boldsymbol{x}\}, & \text { if } \boldsymbol{y}(\cdot) \text { passes through } \boldsymbol{x}, \\ +\infty & \text { otherwise. }\end{cases}
$$

We will often omit the last argument $\tau$ if $\boldsymbol{y}_{0} \in \partial \Omega$ and the starting time is $\tau=q\left(\boldsymbol{y}_{0}\right)$. We define a value function for $\boldsymbol{x} \in \Omega$ as follows:

$$
u(\boldsymbol{x})=\inf _{\boldsymbol{y}_{0} \in \partial \Omega, \boldsymbol{a}(\cdot) \in \mathcal{A}} \mathcal{J}\left(\boldsymbol{x}, \boldsymbol{a}(\cdot), \boldsymbol{y}_{0}\right) .
$$

REMARK 2.1 The min-time problem is among the most classical in control theory. A detailed theoretical discussion and extensive bibliography can be found, for example, in [1]. Here we note several differences between our formulation and the usual setting.

1. The problem is normally formulated for an autonomous dynamical system. To treat our case in that framework, one would have to switch to $\boldsymbol{z}=(\boldsymbol{y}, t)$ or, equivalently, to define a value function $u(\boldsymbol{x}, \tau)$, where $\tau$ is the (re)starting time. Indeed, for the problem considered in Section 4 this cannot be avoided. Here, however, the optimality condition (and the corresponding PDE) can be derived without raising the dimensionality. The existence of efficient non-iterative methods for the static PDE (e.g., [30]) makes such an interpretation preferable from the computational point of view.

2. The assumptions (4) guarantee the continuity of the value function on $\Omega$ even in the presence of state-constraints; $F_{1}>0$ yields both Soner's tangentiality along the boundary of the constraint set (as in [32]) and the local controllability near $\partial \Omega$ (as in [2], for example). This model is admittedly restrictive since many realistic problems in control theory and robotics do not satisfy the small-time controllability requirement. A much more general treatment is possible, but we choose to ignore it to concentrate on the key idea (i.e., derivation of a static PDE for the value function of a non-autonomous problem).

3. As discussed in [30], the Ordered Upwind Method also relies on $F_{1}>0$, but this restriction can often be relaxed in practice. For example, if the value function $u(x)$ is Lipschitz-continuous and 
$L_{u}$ is its Lipschitz constant, then the anisotropy coefficient can be similarly defined as $\Upsilon=L_{u} F_{2}$ even if $F_{1}=0$. (Note that $F_{1}>0$ implies $L_{u} \leqslant F_{1}^{-1}$.) As shown in [35], this is sufficient to produce alternative proofs for Lemmas 2.7 and 2.12, justifying the use of the method described in Section 3 in a more general case.

4. We do not assume convexity of the set $f\left(\boldsymbol{x}, S_{1}, t\right)$, thus the optimal control need not exist (though $u(\boldsymbol{x})$ is still well defined). To simplify the presentation, we will somewhat loosely refer to the optimal controls and trajectories. The corresponding properties can be formulated and proven for the $\epsilon$-suboptimal controls and trajectories as well.

The following lemma is the reason why a static PDE can be derived for this value function.

LEMMA 2.2 Suppose a trajectory defined by (3) passes through $\boldsymbol{x} \in \Omega$. Let $t_{1}$ be such that $q\left(\boldsymbol{y}_{0}\right) \leqslant t_{1} \leqslant \mathcal{J}\left(\boldsymbol{x}, \boldsymbol{a}(\cdot), \boldsymbol{y}_{0}\right)$ and let $\boldsymbol{x}_{1}=\boldsymbol{y}\left(t_{1}\right)$. Define a trajectory

$$
\begin{aligned}
\hat{\boldsymbol{y}}^{\prime}(\hat{t}) & =f(\hat{\boldsymbol{y}}(\hat{t}), \hat{\boldsymbol{a}}(\hat{t}), \hat{t}) \hat{\boldsymbol{a}}(\hat{t}), \\
\hat{\boldsymbol{y}}\left(u\left(\boldsymbol{x}_{1}\right)\right) & =\boldsymbol{x}_{1},
\end{aligned}
$$

where $\hat{\boldsymbol{a}}(\cdot)$ is chosen so that $\hat{\boldsymbol{y}}(\hat{t})=\boldsymbol{y}(t)$. Then

$$
\mathcal{J}\left(\boldsymbol{x}, \hat{\boldsymbol{a}}(\cdot), \boldsymbol{x}_{1}, u\left(\boldsymbol{x}_{1}\right)\right) \leqslant \mathcal{J}\left(\boldsymbol{x}, \boldsymbol{a}(\cdot), \boldsymbol{y}_{0}\right),
$$

and the above inequality becomes strict if $t_{1}>u\left(\boldsymbol{x}_{1}\right)$.

Proof. First, we note that if the speed $f$ were assumed decreasing in time, the lemma would simply follow from the fact that $u\left(\boldsymbol{x}_{1}\right) \leqslant \mathcal{J}\left(\boldsymbol{x}_{1}, \boldsymbol{a}(\cdot), \boldsymbol{y}_{0}\right)=t_{1}$. However, the result holds even if $f$ is not monotone.

Define $\hat{t}$ by

$$
\begin{aligned}
\hat{t}^{\prime}(t) & =\frac{f(\boldsymbol{y}(t), \boldsymbol{a}(t), t)}{f(\boldsymbol{y}(t), \boldsymbol{a}(t), \hat{t})} \quad \text { for } t>t_{1} ; \\
\hat{t}\left(t_{1}\right) & =u\left(\boldsymbol{x}_{1}\right) .
\end{aligned}
$$

Under the assumptions (4), the ODE (8) has a unique and strictly increasing solution for any initial conditions. Since $\hat{t}(t)=t$ also satisfies $(8)$ and $\hat{t}\left(t_{1}\right) \leqslant t_{1}$, it follows that

$$
\hat{t}(t) \leqslant t \quad \text { for all } t>t_{1} .
$$

We also note that, since $\hat{t}(t)$ is strictly increasing, its inverse $t(\hat{t})$ is well defined as long as $\boldsymbol{y}(t) \in \Omega$. For a system defined by

$$
\begin{aligned}
\hat{\boldsymbol{y}}^{\prime}(\hat{t}) & =f(\boldsymbol{y}(t(\hat{t})), \boldsymbol{a}(t(\hat{t})), \hat{t}) \boldsymbol{a}(t(\hat{t})), \\
\hat{\boldsymbol{y}}\left(u\left(\boldsymbol{x}_{1}\right)\right) & =\boldsymbol{x}_{1},
\end{aligned}
$$

we obtain

$$
\frac{\mathrm{d}}{\mathrm{d} t}(\boldsymbol{y}(t)-\hat{\boldsymbol{y}}(\hat{t}))=f(\boldsymbol{y}(t), \boldsymbol{a}(t), t) \boldsymbol{a}(t)-f(\boldsymbol{y}(t), \boldsymbol{a}(t), \hat{t}) \boldsymbol{a}(t(\hat{t})) \hat{t}^{\prime}(t)=0 .
$$

Since $\boldsymbol{y}(t)=\hat{\boldsymbol{y}}(\hat{t})$, the ODE 11 can be rewritten as 7 by setting $\hat{\boldsymbol{a}}(\hat{t})=\boldsymbol{a}(t(\hat{t}))$. Thus,

$$
\mathcal{J}\left(\boldsymbol{x}, \hat{\boldsymbol{a}}(\cdot), \boldsymbol{x}_{1}, u\left(\boldsymbol{x}_{1}\right)\right)=\hat{t}\left(\mathcal{J}\left(\boldsymbol{x}, \boldsymbol{a}(\cdot), \boldsymbol{y}_{0}\right)\right) \leqslant \mathcal{J}\left(\boldsymbol{x}, \boldsymbol{a}(\cdot), \boldsymbol{y}_{0}\right)
$$


Finally, if $u\left(x_{1}\right)<t_{1}$, then the non-intersection of integral curves of the ODE (8) guarantees that $\hat{t}(t)<t$ for all $t>t_{1}$, yielding

$$
\mathcal{J}\left(\boldsymbol{x}, \hat{\boldsymbol{a}}(\cdot), \boldsymbol{x}_{1}, u\left(\boldsymbol{x}_{1}\right)\right)=\hat{t}\left(\mathcal{J}\left(\boldsymbol{x}, \boldsymbol{a}(\cdot), \boldsymbol{y}_{0}\right)\right)<\mathcal{J}\left(\boldsymbol{x}, \boldsymbol{a}(\cdot), \boldsymbol{y}_{0}\right) .
$$

COROLlaRY 2.3 If $\boldsymbol{y}(\cdot)$ defined by (3) is a time-optimal trajectory to reach $\boldsymbol{x}$ from the boundary, then $u\left(\boldsymbol{y}\left(t_{1}\right)\right)=t_{1}$ for all $t_{1} \in\left(q\left(\boldsymbol{y}_{0}\right), u(\boldsymbol{x})\right)$.

Proof. First, $u\left(\boldsymbol{y}\left(t_{1}\right)\right) \leqslant t_{1}$ simply by the definition of the value function. If $u\left(\boldsymbol{x}_{1}\right)<t_{1}$ for some $\boldsymbol{x}_{1}=\boldsymbol{y}\left(t_{1}\right)$, then a strictly better trajectory to reach $\boldsymbol{x}$ could be constructed by using an optimal trajectory to reach $\boldsymbol{x}_{1}$ and then continuing via $\hat{\boldsymbol{y}}(\cdot)$ as described in Lemma 2.2. This would contradict the assumption that $\boldsymbol{y}(\cdot)$ is time-optimal.

The following two versions of the optimality principle can be easily proven using the above lemma.

Lemma 2.4 (Fixed-Time Optimality Principle) Let $d(\boldsymbol{x})$ be the minimum distance to the boundary $\partial \Omega$. Then for every point $\boldsymbol{x} \in \Omega$ and for any $\Delta t<d(\boldsymbol{x}) / F_{2}$,

$$
u(\boldsymbol{x})=\inf _{\boldsymbol{y}_{0} \in \partial \Omega, \boldsymbol{a}(\cdot) \in \mathcal{A}}\left\{\Delta t+u\left(\boldsymbol{y}\left(\mathcal{J}\left(\boldsymbol{x}, \boldsymbol{a}(\cdot), \boldsymbol{y}_{0}\right)-\Delta t\right)\right)\right\},
$$

where $\boldsymbol{y}(\cdot)$ is a trajectory passing through the point $\boldsymbol{x}$ and corresponding to a chosen control $\boldsymbol{a}(\cdot)$.

Lemma 2.5 (Fixed-Boundary Optimality Principle) Consider an open connected set $\Omega_{1} \subset \Omega$ and an arbitrary point $\boldsymbol{x} \in \Omega_{1}$. Then

$$
u(\boldsymbol{x})=\inf _{\boldsymbol{y}_{0} \in \partial \Omega_{1}, \boldsymbol{a}(\cdot) \in \mathcal{A}} \mathcal{J}\left(\boldsymbol{x}, \boldsymbol{a}(\cdot), \boldsymbol{y}_{0}, u\left(\boldsymbol{y}_{0}\right)\right)
$$

We now enumerate several properties of the value function $u(x)$ (the proofs are similar to those for the time-optimal autonomous control in [30]).

LEMma 2.6 The value function $u(x)$ is Lipschitz-continuous and bounded on $\Omega$. If $\boldsymbol{y}^{\prime}(t)=$ $f(\boldsymbol{y}(t), \boldsymbol{a}(t), t) \boldsymbol{a}(t)$ defines an optimal trajectory to a point $\boldsymbol{x}$ (i.e., $\left.u(\boldsymbol{x})=\mathcal{J}\left(\boldsymbol{x}, \boldsymbol{a}(\cdot), \boldsymbol{y}_{0}\right)\right)$, then the function $u(\boldsymbol{y}(t))$ is strictly increasing for $t \in\left[q\left(\boldsymbol{y}_{0}\right), u(\boldsymbol{x})\right]$.

Lemma 2.7 Consider a point $\overline{\boldsymbol{x}} \in \Omega$. Then, for any constant $C$ such that $q_{2} \leqslant C \leqslant u(\overline{\boldsymbol{x}})$, the optimal trajectory for $\overline{\boldsymbol{x}}$ will intersect the level set $u(\boldsymbol{x})=C$ at some point $\tilde{\boldsymbol{x}}$. If $\overline{\boldsymbol{x}}$ is distance $d_{1}$ away from that level set, then

$$
\|\tilde{\boldsymbol{x}}-\overline{\boldsymbol{x}}\| \leqslant d_{1} \Upsilon .
$$

Proof. Briefly, if $\tilde{\boldsymbol{x}}$ is too far, then $\overline{\boldsymbol{x}}$ can be reached more quickly starting from $\hat{\boldsymbol{x}}$, the closest point to $\overline{\boldsymbol{x}}$ such that $u(\hat{\boldsymbol{x}})=C$.

REMARK 2.8 A better (but implicit) bound is

$$
\|\tilde{\boldsymbol{x}}-\overline{\boldsymbol{x}}\| \leqslant d_{1} \Upsilon_{\|\tilde{\boldsymbol{x}}-\overline{\boldsymbol{x}}\|}(\overline{\boldsymbol{x}}) .
$$

An explicit approximation of the above can be derived recursively:

$$
\|\tilde{\boldsymbol{x}}-\overline{\boldsymbol{x}}\| \leqslant d_{1} K,
$$

where $K$ is the limit of the non-increasing sequence

$$
K_{0}=\Upsilon, \quad K_{n+1}=\Upsilon_{d_{1} K_{n}}(\overline{\boldsymbol{x}}) .
$$

An additional improvement is achieved by noting that, in computing the anisotropy coefficient, $f_{1}(\boldsymbol{y})$ can be minimized in $B_{d_{1}}$ instead of $B_{d_{1} K_{n}}$. 
LEMMA 2.9 Consider an unstructured (triangulated) mesh $X$ of diameter $h$ on a simply-connected domain $\Omega$. Consider a simple closed curve $\Gamma \subset \Omega$ and suppose it is "well-resolved" by $X$, i.e., for any point $\boldsymbol{x}$ on $\Gamma$, there exists a mesh point $\hat{\boldsymbol{x}}$ inside $\Gamma$ such that $\|\boldsymbol{x}-\hat{\boldsymbol{x}}\|<h$. Suppose $\overline{\boldsymbol{x}}$ is a mesh point inside $\Gamma$ such that $u(\overline{\boldsymbol{x}}) \leqslant u\left(\boldsymbol{x}_{i}\right)$ for all other mesh points $\boldsymbol{x}_{i} \in X$ inside $\Gamma$. Then the optimal trajectory for $\overline{\boldsymbol{x}}$ will intersect $\Gamma$ at some point $\tilde{\boldsymbol{x}}$ such that

$$
\|\tilde{\boldsymbol{x}}-\overline{\boldsymbol{x}}\| \leqslant h \Upsilon .
$$

Proof. Briefly, if $\overline{\boldsymbol{x}}$ is too far from $\tilde{\boldsymbol{x}} \in \Gamma$, then reaching from $\tilde{\boldsymbol{x}}$ a nearby mesh point $\hat{\boldsymbol{x}}$ inside $\Gamma$ will take less time than it takes to reach $\overline{\boldsymbol{x}}$. This yields $u(\overline{\boldsymbol{x}})>u(\hat{\boldsymbol{x}})$, a contradiction.

REMARK 2.10 The assumption that $\Omega$ is simply-connected is only used to guarantee that an optimal trajectory for $\overline{\boldsymbol{x}}$ will intersect $\Gamma$.

In addition, a tighter implicit bound can be obtained by replacing $\Upsilon$ by $\Upsilon_{R}(\hat{\boldsymbol{x}})$ for $R=$ $\|\tilde{\boldsymbol{x}}-\overline{\boldsymbol{x}}\|+h$. A recursive formula can be written by using the limit of the non-increasing sequence

$$
K_{0}=\Upsilon, \quad K_{n+1}=\Upsilon_{h\left(1+K_{n}\right)}(\overline{\boldsymbol{x}}) .
$$

An infinitesimal version can be formally derived for the Optimality Principle (12), i.e., it is easy to show that, wherever $\nabla u$ is well-defined, $u$ is a classical solution of the Hamilton-Jacobi-Bellman PDE

$$
\begin{aligned}
\max _{\boldsymbol{a} \in S_{1}}\{(\nabla u(\boldsymbol{x}) \cdot \boldsymbol{a}) f(\boldsymbol{x}, \boldsymbol{a}, u(\boldsymbol{x}))\} & =1, & & \boldsymbol{x} \in \Omega, \\
u(\boldsymbol{x}) & =q(\boldsymbol{x}), & & \boldsymbol{x} \in \partial \Omega .
\end{aligned}
$$

This PDE can be rewritten in the form

$$
H(\nabla u, u, \boldsymbol{x})=\|\nabla u(x)\| F\left(\boldsymbol{x}, \frac{\nabla u(\boldsymbol{x})}{\|\nabla u(\boldsymbol{x})\|}, u(\boldsymbol{x})\right)=1,
$$

where $F$ is a homogeneous Legendre transform of the speed $f$ :

$$
F(\boldsymbol{x}, \boldsymbol{n}, t)=\max _{\boldsymbol{a} \in S_{1}}\{(\boldsymbol{n} \cdot \boldsymbol{a}) f(\boldsymbol{x}, \boldsymbol{a}, t)\} .
$$

A dual interpretation describes $F$ as a "normal speed" of an anisotropically contracting front, whose position at time $t$ is the level set $u(\boldsymbol{x})=t$. (See [10] and [33] for a detailed treatment of the case $F=F(\boldsymbol{x}, \boldsymbol{n})$.) Here we simply note that the Hamiltonian $H$ is Lipschitz-continuous, convex, and homogeneous of degree one in the first argument.

If an optimal trajectory to a point $\boldsymbol{x}$ exists, then it can be easily shown to coincide with a characteristic of (16) passing through that point. If an optimal trajectory is not unique, then several characteristics collide at $\boldsymbol{x}$ and $\nabla u(\boldsymbol{x})$ is undefined. Thus, (16) usually does not have a smooth solution, and a weak (Lipschitz-continuous) solution is not unique. The uniqueness is achieved by defining a viscosity solution [8], imposing additional conditions on smooth test functions [7] as follows:

A bounded, uniformly continuous function $u$ is a viscosity solution of (16) if the following holds for each smooth test function $\phi \in C_{c}^{\infty}(\Omega)$ :

(i) if $u-\phi$ has a local minimum at $\boldsymbol{x}_{0} \in \Omega$ then

$$
\max _{\boldsymbol{a} \in S_{1}}\left\{\left(\nabla \phi\left(\boldsymbol{x}_{0}\right) \cdot \boldsymbol{a}\right) f\left(\boldsymbol{x}_{0}, \boldsymbol{a}, u\left(\boldsymbol{x}_{0}\right)\right)\right\}-1 \geqslant 0 ;
$$

(ii) if $u-\phi$ has a local maximum at $x_{0} \in \Omega$ then

$$
\max _{\boldsymbol{a} \in S_{1}}\left\{\left(\nabla \phi\left(\boldsymbol{x}_{0}\right) \cdot \boldsymbol{a}\right) f\left(\boldsymbol{x}_{0}, \boldsymbol{a}, u\left(\boldsymbol{x}_{0}\right)\right)\right\}-1 \leqslant 0 .
$$


The Optimality Principle can be used to show that the value function is a viscosity solution. We omit this proof here since the argument is virtually the same as for the autonomous control (see, for example, [1, Chapter 4.2]). On the other hand, a usual proof of uniqueness assumes the monotonicity of the Hamiltonian in $u$. We now show that this assumption is unnecessary in our setting.

LEmma 2.11 There exists only one non-negative, bounded viscosity solution $u(x)$ of equation $(16)$.

Proof. Let $u_{\max }=\max _{\Omega} u(x)$ and use $L_{H}$ to denote the Lipschitz constant for the Hamiltonian (17).

Employing a Kruzhkov-type transform [19], we define $v(\boldsymbol{x})=\lambda^{-1}\left(1-e^{-u(\boldsymbol{x})}\right)$, where $\lambda$ is chosen so that $0<\lambda<\left(e^{u_{\max }} L_{H}\right)^{-1}$. Then

$$
v(\boldsymbol{x})<r_{\max }=\lambda^{-1}\left(1-e^{-u_{\max }}\right), \quad u(\boldsymbol{x})=-\log (1-\lambda v(\boldsymbol{x})), \quad \nabla u=\frac{\lambda}{1-\lambda v} \nabla v .
$$

Define a new Hamiltonian

$$
\widetilde{H}(\boldsymbol{p}, r, \boldsymbol{x})=r+\max _{\boldsymbol{a} \in S_{1}}\{(\boldsymbol{p} \cdot \boldsymbol{a}) f(\boldsymbol{x}, \boldsymbol{a},-\log (1-\lambda r))\} .
$$

By a standard argument (see, for example, [1, Proposition 2.2.5]), $v$ is a viscosity solution of

$$
\begin{aligned}
\widetilde{H}(\nabla v(\boldsymbol{x}), v(\boldsymbol{x}), \boldsymbol{x}) & =\lambda^{-1}, & & \boldsymbol{x} \in \Omega, \\
v(\boldsymbol{x}) & =\lambda^{-1}\left(1-e^{-q(\boldsymbol{x})}\right), & & \boldsymbol{x} \in \partial \Omega,
\end{aligned}
$$

if and only if $u$ is a viscosity solution of (16).

For $0 \leqslant r_{1}<r_{2} \leqslant r_{\max }$,

$$
\begin{aligned}
\widetilde{H}\left(\boldsymbol{p}, r_{2}, \boldsymbol{x}\right)-\widetilde{H}\left(\boldsymbol{p}, r_{1}, \boldsymbol{x}\right) & \geqslant\left(r_{2}-r_{1}\right)-L_{H}\left(\log \left(1-\lambda r_{1}\right)-\log \left(1-\lambda r_{2}\right)\right) \\
= & \left(r_{2}-r_{1}\right)-L_{H}\left(r_{2}-r_{1}\right) \frac{\lambda}{1-\lambda r^{*}}=\left(r_{2}-r_{1}\right)-L_{H}\left(r_{2}-r_{1}\right) \lambda e^{u^{*}},
\end{aligned}
$$

for some $r^{*} \in\left[r_{1}, r_{2}\right]$ and for the corresponding $u^{*} \leqslant u_{\max }$. By the definition of $\lambda, C=1-$ $L_{H} \lambda e^{u_{\max }}>0$, and

$$
\widetilde{H}\left(\boldsymbol{p}, r_{2}, \boldsymbol{x}\right)-\widetilde{H}\left(\boldsymbol{p}, r_{1}, \boldsymbol{x}\right)>C\left(r_{2}-r_{1}\right)>0 .
$$

Hence, $\widetilde{H}(\boldsymbol{p}, r, \boldsymbol{x})$ is monotone in $r$ for $r \in\left[0, r_{\max }\right]$, and a standard argument (e.g., [1, Chapter 2.3]) guarantees the uniqueness of thus bounded viscosity solution for (22). This, in turn, implies the uniqueness of the non-negative viscosity solution bounded by $u_{\max }$ for 16 .

We now use the fact that $F_{1}$ is positive to modify the usual definition of the viscosity solution. This idea is fundamental for the numerical methods of Section 3.

Lemma 2.12 Define $S_{1}^{\phi, \boldsymbol{x}}=\left\{\boldsymbol{a} \in S_{1} \mid \boldsymbol{a} \cdot \nabla \phi(\boldsymbol{x}) \geqslant\|\nabla \phi(\boldsymbol{x})\|(\Upsilon(\boldsymbol{x}))^{-1}\right\}$. An equivalent definition of the viscosity solution for 16 can be obtained by using $S_{1}^{\phi, \boldsymbol{x}_{0}}$ instead of $S_{1}$ in the inequalities (19) and (20).

Proof. We first observe that, since $f \geqslant F_{1}>0$, if the maximum is attained for some $\boldsymbol{a}=\boldsymbol{a}_{1}$, then $\boldsymbol{a}_{1} \cdot \nabla \phi\left(\boldsymbol{x}_{0}\right)>0$. Let $\boldsymbol{b}=\nabla \phi\left(\boldsymbol{x}_{0}\right) /\left\|\nabla \phi\left(\boldsymbol{x}_{0}\right)\right\|$. Since $\boldsymbol{a}_{1}$ is a maximizer, we have

$$
\left(\nabla \phi\left(\boldsymbol{x}_{0}\right) \cdot \boldsymbol{a}_{1}\right) f\left(\boldsymbol{x}_{0}, \boldsymbol{a}_{1}, u\left(\boldsymbol{x}_{0}\right)\right) \geqslant\left(\nabla \phi\left(\boldsymbol{x}_{0}\right) \cdot \boldsymbol{b}\right) f\left(\boldsymbol{x}_{0}, \boldsymbol{b}, u\left(\boldsymbol{x}_{0}\right)\right) \geqslant\left\|\nabla \phi\left(\boldsymbol{x}_{0}\right)\right\| f_{1}\left(\boldsymbol{x}_{0}\right) .
$$


Therefore,

$$
\boldsymbol{a}_{1} \cdot \nabla \phi\left(\boldsymbol{x}_{0}\right) \geqslant\left\|\nabla \phi\left(\boldsymbol{x}_{0}\right)\right\| \frac{f_{1}\left(\boldsymbol{x}_{0}\right)}{f\left(\boldsymbol{x}_{0}, \boldsymbol{a}_{1}, u\left(\boldsymbol{x}_{0}\right)\right)} \geqslant\left\|\nabla \phi\left(\boldsymbol{x}_{0}\right)\right\|\left(\Upsilon\left(\boldsymbol{x}_{0}\right)\right)^{-1}
$$

REMARK 2.13 The above lemma practically establishes an upper bound on the angle between the characteristic of the PDE (16) and the gradient line of its viscosity solution. Indeed, if $\nabla u\left(x_{0}\right)$ is well-defined, then $\nabla u\left(\boldsymbol{x}_{0}\right)=\nabla \phi\left(\boldsymbol{x}_{0}\right)$, and $\boldsymbol{a}_{1} \cdot \nabla u\left(\boldsymbol{x}_{0}\right) \geqslant\left\|\nabla u\left(\boldsymbol{x}_{0}\right)\right\| \Upsilon^{-1}\left(\boldsymbol{x}_{0}\right)$. If $\gamma$ is the angle between $\nabla u\left(x_{0}\right)$ and $\boldsymbol{a}_{1}$, then $\cos (\gamma) \geqslant 1 / \Upsilon\left(\boldsymbol{x}_{0}\right)$. We note that this result is consistent with what was derived for the case of autonomous control in [30] with one caveat: since the problem considered in [30] is a min-time-from-boundary, a similar upper bound is established there on the angle between $\nabla u\left(\boldsymbol{x}_{0}\right)$ and $-\boldsymbol{a}_{1}$.

Finally, we observe that the problem considered in this section can be alternatively interpreted as an implicitly defined autonomous control problem. In particular, supposing that $u(\boldsymbol{x})$ is a known value function for the problem (3), we could define the new dynamics by

$$
\begin{aligned}
\boldsymbol{y}^{\prime}(t) & =f(\boldsymbol{y}(t), \boldsymbol{a}(t), u(\boldsymbol{y}(t))) \boldsymbol{a}(t), \\
\boldsymbol{y}\left(\tau=q\left(\boldsymbol{y}_{0}\right)\right) & =\boldsymbol{y}_{0} \in \partial \Omega,
\end{aligned}
$$

and let $u^{*}(\boldsymbol{x})$ be the value function for this system. It is easy to see that $u^{*}=u$ from the uniqueness of the viscosity solution to the PDE

$$
\left\|\nabla u^{*}(\boldsymbol{x})\right\| F\left(\boldsymbol{x}, \frac{\nabla u^{*}(\boldsymbol{x})}{\left\|\nabla u^{*}(\boldsymbol{x})\right\|}, u(\boldsymbol{x})\right)=1
$$

corresponding to the system (23). However, Corollary 2.3 yields a more precise result: the characteristics of the PDEs (16) and 24) also coincide. This is a much stronger relation. For example, the viscosity solution $u^{* *}$ of the eikonal PDE

$$
\left\|\nabla u^{* *}(\boldsymbol{x})\right\| F\left(\boldsymbol{x}, \frac{\nabla u(\boldsymbol{x})}{\|\nabla u(\boldsymbol{x})\|}, u(\boldsymbol{x})\right)=1
$$

also coincides with $u(\boldsymbol{x})$, but the characteristics of this PDE are generally quite different (in fact, for this PDE they coincide with the gradient lines of $u(x))$.

\section{Min-time-from-boundary: Ordered Upwind Method}

The non-iterative method presented below is a generalization of the semi-Lagrangian OUM for the autonomous control introduced in [29]; a detailed discussion of earlier Dijkstra-like methods for the eikonal case can also be found in [30].

OUMs are typically defined on an unstructured mesh $X$ discretizing $\bar{\Omega}$. We refer to a pair of mesh points $\boldsymbol{x}_{j}, \boldsymbol{x}_{k}$ as "adjacent" whenever both of them are vertices of the same triangle in $X$. We assume that the mesh $X$ is such that

- the mesh-diameter is $h$, i.e., if mesh points $\boldsymbol{x}_{j}$ and $\boldsymbol{x}_{k}$ are adjacent then $\left\|\boldsymbol{x}_{j}-\boldsymbol{x}_{k}\right\| \leqslant h$;

- $\partial \Omega$ is "well-resolved", i.e., for each $\boldsymbol{y} \in \partial \Omega$ there exists a mesh point $\boldsymbol{x}_{i} \in B_{h}(\boldsymbol{y}) \cap \partial \Omega$. 
For notational convenience, we define the "mesh degeneracy" coefficient $\eta=h / h_{\min }$, where $h_{\min }$ is the smallest triangle height in $X$.

The general idea behind OUMs is to allow the space-marching for the boundary value problems - not unlike explicit forward-time marching for initial-boundary value problems. The solution can be "marched" (on the mesh) from the boundary using the characteristic information, and a new (smaller) boundary value problem can be posed using the newly computed "boundary"the current divide between the already-computed (Accepted) and not-yet-touched (Far) mesh points. The mesh discretization of that "new boundary" is referred to as AcceptedFront; the notyet-Accepted mesh points which are adjacent to the AcceptedFront are designated Considered. A tentative value $(V(\boldsymbol{x}))$ can be computed for each Considered mesh point $\boldsymbol{x}$ under the assumption that its characteristic intersects the AcceptedFront in some vicinity of that mesh point (designated $N F(\boldsymbol{x})$ ). All Considered points are sorted by their tentative values $V$, and a typical step of the algorithm consists of picking the Considered $\bar{x}$ with the smallest $V$ and making it Accepted $(U(\overline{\boldsymbol{x}}):=V(\overline{\boldsymbol{x}}))$. This operation modifies the AcceptedFront $(\overline{\boldsymbol{x}}$ in, other mesh points possibly out), and causes a possible recomputation of all the not-yet-Accepted mesh-points near $\overline{\boldsymbol{x}}$.

We note that Lemma 2.9 provides a practical upper bound on a distance between the smallest Considered mesh point $\bar{x}$ and the intersection of its characteristic with the AcceptedFront; see also Figure 1. Furthermore, we can use $h \Upsilon_{d}(\boldsymbol{x})$ for any positive fixed $d>h \Upsilon$; see also Remark 2.10

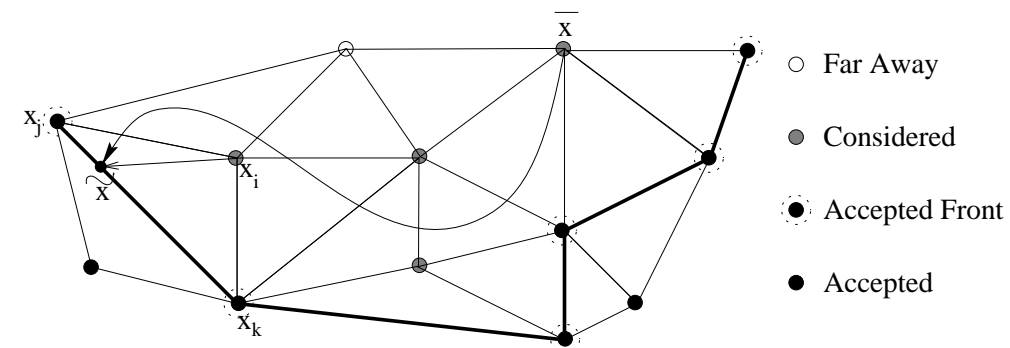

FIG. 1. The AcceptedFront and the Considered mesh points. Segments of $A F$ are shown in bold. The optimal trajectory to $\overline{\boldsymbol{x}}$ cannot intersect $A F$ too far away from $\overline{\boldsymbol{x}}$, for if $\|\tilde{\boldsymbol{x}}-\overline{\boldsymbol{x}}\|>h \Upsilon$, then $u\left(\boldsymbol{x}_{i}\right)<u(\overline{\boldsymbol{x}})$.

Given a pair of adjacent AcceptedFront mesh points $\boldsymbol{x}_{j}, \boldsymbol{x}_{k}$, we define an update formula for $V(\boldsymbol{x})$ under the assumption that an optimal trajectory to $\boldsymbol{x}$ intersects $\boldsymbol{x}_{j} \boldsymbol{x}_{k}$ at some point $\tilde{\boldsymbol{x}}$ and then follows a straight line to $\boldsymbol{x}$ :

$$
\begin{aligned}
\tilde{\boldsymbol{x}}_{\zeta} & =\zeta \boldsymbol{x}_{j}+(1-\zeta) \boldsymbol{x}_{k} ; \\
\Delta(\zeta) & =\left\|\tilde{\boldsymbol{x}}_{\zeta}-\boldsymbol{x}\right\| ; \\
\boldsymbol{a}_{\zeta} & =\frac{\boldsymbol{x}-\tilde{\boldsymbol{x}}_{\zeta}}{\Delta(\zeta)} ; \\
U\left(\tilde{\boldsymbol{x}}_{\zeta}\right) & =\zeta U\left(\boldsymbol{x}_{j}\right)+(1-\zeta) U\left(\boldsymbol{x}_{k}\right) ; \\
V_{\boldsymbol{x}_{j} \boldsymbol{x}_{k}} & =\min _{\zeta \in[0,1]}\left\{\frac{\Delta(\zeta)}{f\left(\boldsymbol{x}, \boldsymbol{a}_{\zeta}, U\left(\tilde{\boldsymbol{x}}_{\zeta}\right)\right)}+U\left(\tilde{\boldsymbol{x}}_{\zeta}\right)\right\} .
\end{aligned}
$$

This semi-Lagrangian discretization is related to the numerical schemes developed in [20], [21], and [14]. However, we emphasize that $V_{\boldsymbol{x}_{j} \boldsymbol{x}_{k}}(\boldsymbol{x})$ is defined even if $\boldsymbol{x}_{j}$ and $\boldsymbol{x}_{k}$ are not adjacent to $\boldsymbol{x}$. 
Assuming the existence of a locally smooth (sub)optimal trajectory and the smoothness of the value function on the segment $\boldsymbol{x}_{j} \boldsymbol{x}_{k}$, it is easy to show that the local truncation error is $O\left(h^{2}\right)$. The first of these assumptions is satisfied if the set $f\left(\boldsymbol{x}, S_{1}, t\right)$ is convex for all $\boldsymbol{x} \in \bar{\Omega}, t \in \mathbb{R}_{+, 0}$; see [1]. The second assumption might be wrong near the shocks (where $\nabla u$ is discontinuous), but this does not affect the global convergence since the characteristics might run into a shock, but never originate from the shock [30].

We now briefly state the algorithm and refer to [30] for the implementation details and possible optimizations.

\section{Semi-Lagrangian Ordered Upwind Method}

All the nodes are divided into three classes: Far (no information about the correct value of $U$ is known), Accepted (the correct value of $U$ has been computed), and Considered (adjacent to Accepted). The AcceptedFront is defined as a set of Accepted mesh points, which are adjacent to some not-yet-accepted (i.e., Considered) mesh points. Define the set $A F$ of the line segments $\boldsymbol{x}_{j} \boldsymbol{x}_{k}$, where $\boldsymbol{x}_{j}$ and $\boldsymbol{x}_{k}$ are adjacent mesh points on the AcceptedFront, such that there exists a Considered mesh point $\boldsymbol{x}_{i}$ adjacent to both $\boldsymbol{x}_{j}$ and $\boldsymbol{x}_{k}$. For each Considered mesh point $\boldsymbol{x}$ we define the "near front" as the part of $A F$ "relevant to $x$ ":

$$
\mathrm{NF}(\boldsymbol{x})=\left\{\boldsymbol{x}_{j} \boldsymbol{x}_{k} \in A F \mid \exists \tilde{\boldsymbol{x}} \text { on } \boldsymbol{x}_{j} \boldsymbol{x}_{k} \text { such that }\|\tilde{\boldsymbol{x}}-\boldsymbol{x}\| \leqslant h \Upsilon_{d}(\boldsymbol{x})\right\}
$$

1. Start with all the mesh points in Far.

2. Move the mesh points on the boundary $(\boldsymbol{y} \in \partial \Omega)$ to Accepted $(U(\boldsymbol{y}):=q(\boldsymbol{y}))$.

3. Move all the mesh points $\boldsymbol{x}$ adjacent to the boundary into Considered and evaluate the tentative values

$$
V(\boldsymbol{x}):=\min _{\boldsymbol{x}_{j} \boldsymbol{x}_{k} \in \mathrm{NF}(\boldsymbol{x})} V_{\boldsymbol{x}_{j}, \boldsymbol{x}_{k}}(\boldsymbol{x}) .
$$

4. Find the mesh point $\overline{\boldsymbol{x}}$ with the smallest value of $V$ among all the Considered.

5. Move $\overline{\boldsymbol{x}}$ to Accepted $(U(\overline{\boldsymbol{x}}):=V(\overline{\boldsymbol{x}}))$ and update the AcceptedFront.

6. Move the Far mesh points adjacent to $\bar{x}$ into Considered and compute their tentative values by (26).

7. Recompute the value for all the other Considered $\boldsymbol{x}$ such that $\bar{x} \in \mathrm{NF}(\boldsymbol{x})$ :

$$
V(\boldsymbol{x}):=\min \left\{V(\boldsymbol{x}), \min _{\overline{\boldsymbol{x}} \boldsymbol{x}_{i} \in \mathrm{NF}(\boldsymbol{x})} V_{\overline{\boldsymbol{x}}, \boldsymbol{x}_{i}}(\boldsymbol{x})\right\} .
$$

8. If Considered is not empty then go to 4.

Efficiency. This results in a "single-pass" method since the maximum number of times each mesh point can be re-evaluated is bounded by the number of mesh points in the $h \Upsilon_{d}$ neighborhood of that point. Thus, this method formally has the computational complexity of $O\left(\Upsilon^{2} M \log (M)\right)$. Moreover, since the AcceptedFront is approximating the level set of the viscosity solution $u$, as the mesh is refined, the complexity will behave as $O(\Upsilon M \log (M))$. The above computational complexity bounds are derived for the implementation that uses heap-sort data structures [30].

Convergence. The proof of the following convergence result is omitted since it is virtually the same as for the autonomous case [30]: 
PROPOSITION 3.1 Let $\left\{X_{r}\right\}$ be a sequence of meshes and $\left\{U^{r}\right\}$ be a sequence of approximate solutions obtained on $X_{r}$ as described above. If $\left\{X_{r}\right\}$ is such that $\partial \Omega$ remains "well-resolved", $h_{r} \rightarrow 0$, and $\eta_{r}<\eta$ as $r \rightarrow \infty$, then $U^{r}$ uniformly converge to the viscosity solution of (16) as $r \rightarrow \infty$.

The numerical evidence indicates that the $L_{\infty}$ error decreases as $O(h)$; this corresponds to the accuracy of the update formula $(25)$ and the piecewise-smoothness of optimal trajectories in the examples considered.

\subsection{Min-time-from-boundary: numerical examples}

We consider a "min-time control from a point" problem with the state constraint $\boldsymbol{y}(t) \in Q=$ $(0,100) \times(0,100)$. We let $\Omega=Q \backslash\left\{\left(x_{0}, y_{0}\right)\right\}$ and select $q\left(x_{0}, y_{0}\right)=0$, and $q=+\infty$ on $\partial Q$. A tourist is moving through a rough landscape modeled by the graph of

$$
g(x, y)=45 \sin \left(\frac{\pi x}{50}\right) \sin \left(\frac{\pi y}{50}\right), \quad(x, y) \in[0,100] \times[0,100] .
$$

Our goal is to compute the min-time for him to reach each point $(x, y, g(x, y))$ starting from $\left(x_{0}, y_{0}, g\left(x_{0}, y_{0}\right)\right)$; see Figure 2
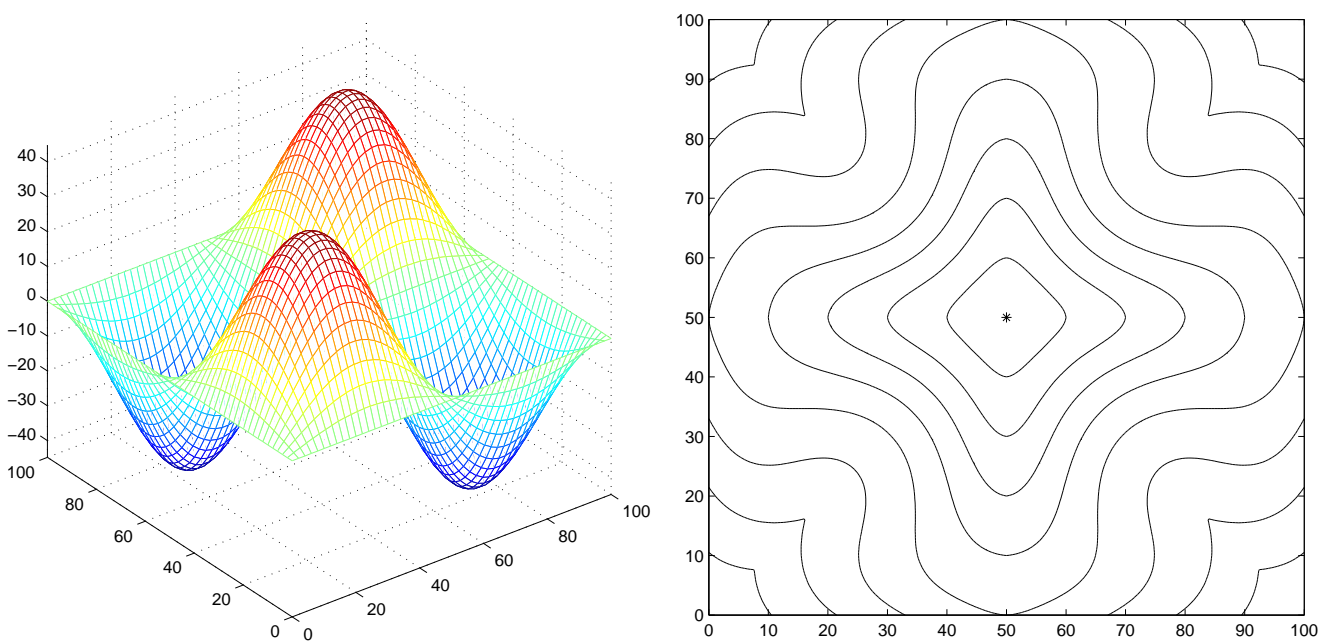

FIG. 2. The test surface (left) and the geodesic circles on that surface projected onto the $x y$ plane (right).

We will further assume that the tourist's speed on the surface is affected by the inclination of that surface along his trajectory. Given a direction of motion $a \in S_{1}$ for the tourist's projection in the $x y$ plane, we can express the angle $\theta$ between the direction of motion on the surface and the positive direction of $z$-axis:

$$
\theta_{\boldsymbol{a}}=\frac{\pi}{2}-\arctan (\nabla g(x, y) \cdot \boldsymbol{a})
$$

If $\phi\left(\theta_{\boldsymbol{a}}\right)$ is an inclination-factor and $\psi(t)$ is a time-factor, then the resulting formula for the speed of tourist's projection in the plane is

$$
f(x, y, \boldsymbol{a}, t)=\phi\left(\theta_{\boldsymbol{a}}\right) \psi(t)\left(1+(\nabla g(x, y) \cdot \boldsymbol{a})^{2}\right)^{-1 / 2} .
$$


In the following experiments we have chosen

$$
\phi(\theta)=\sin ^{6}(\theta)+0.1
$$
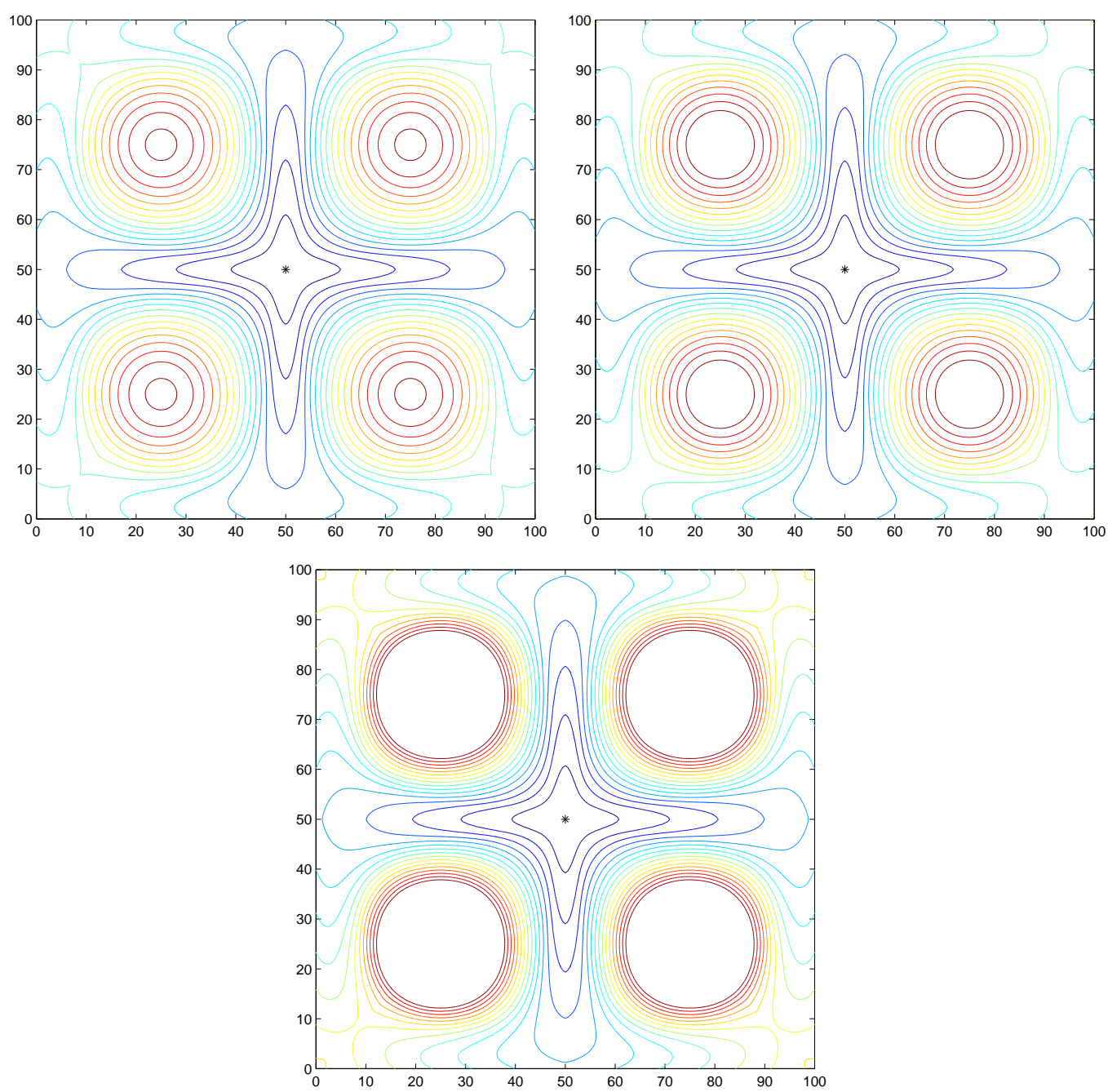

FIG. 3. The min-time-from- $\left(x_{0}, y_{0}\right)$ function for $\psi(t)=e^{-\lambda t}: \lambda=0.0$ (top left), $\lambda=0.001$ (top right), and $\lambda=0.005$ (bottom). The level sets are plotted at the same values in all three cases. The point $\left(x_{0}, y_{0}\right)$ is indicated by an asterisk.

Figure 3 shows the level sets of the value function for a time-monotone case ${ }^{2}$

$$
\psi(t)=e^{-\lambda t}
$$

2 The first example (Figure 3 was previously numerically studied in [31]. 

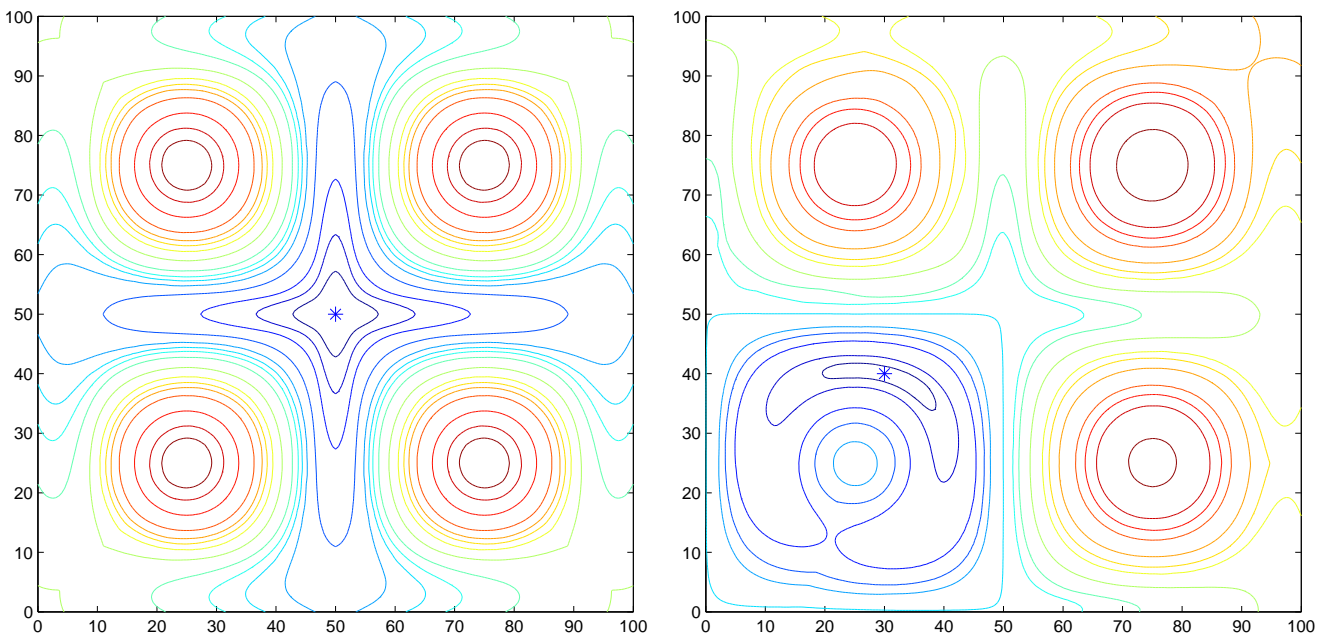

FIG. 4. The min-time-from- $\left(x_{0}, y_{0}\right)$ function for a periodic $\psi(t)$.

and for $\left(x_{0}, y_{0}\right)=(50,50)$. The time-dependence here can be interpreted as an impact of the tourist's tiredness on his ability to move.

Our second example uses an oscillating

$$
\psi(t)=\left[1+\frac{1}{2} \sin \left(\frac{t \pi}{20}\right)\right]^{-1},
$$

thus modeling the dependence on some time-periodic factors (e.g., weather, mood-swings, etc). Figure 4 shows the level sets of the value function for two different choices of $\left(x_{0}, y_{0}\right)$.

\section{Time-dependent case: min-time to the boundary}

Suppose the dynamics on an open bounded domain $\Omega \subset \mathbb{R}^{2}$ is given by

$$
\begin{aligned}
\boldsymbol{y}^{\prime}(s) & =\hat{f}(\boldsymbol{y}(s), \boldsymbol{a}(s), s) \boldsymbol{a}(s), \\
\boldsymbol{y}(t) & =\boldsymbol{x} \in \Omega,
\end{aligned}
$$

where $\boldsymbol{x}$ is the starting point, $t$ is the starting time, $\boldsymbol{a}(\cdot) \in \mathcal{A}$ is the control, and $\hat{f}(\boldsymbol{y}, \boldsymbol{a}, t)$ is the speed of moving through $\boldsymbol{y}$ in the direction $\boldsymbol{a}$ at the time $t$. As in the autonomous case, we define the time-to-boundary as

$$
T(\boldsymbol{x}, \boldsymbol{a}(\cdot), t)=\inf \{s \geqslant t \mid \boldsymbol{y}(s) \in \partial \Omega\}
$$

and the total time-cost associated with using $\boldsymbol{a}(\cdot)$ as

$$
\widehat{\mathcal{J}}(\boldsymbol{x}, \boldsymbol{a}(\cdot), t)=T(\boldsymbol{x}, \boldsymbol{a}(\cdot), t)+\hat{q}(\boldsymbol{y}(T(\boldsymbol{x}, \boldsymbol{a}(\cdot), t)), T(\boldsymbol{x}, \boldsymbol{a}(\cdot), t)),
$$

where $\hat{q}: \partial \Omega \times \mathbb{R} \rightarrow \mathbb{R}_{+, 0}$ is the exit time-penalty. 
The value function for this problem has to be time-dependent as well:

$$
v(\boldsymbol{x}, t)=\inf _{\boldsymbol{a}(\cdot)} \widehat{\mathcal{J}}(\boldsymbol{x}, \boldsymbol{a}(\cdot), t),
$$

since $\tilde{v}(\boldsymbol{x})=v\left(\boldsymbol{x}, t_{0}\right)$ is generally insufficient to formulate an Optimality Principle 3

To mirror the min-time-from-boundary problem, we will again assume that $\hat{f}$ and $\hat{q}$ are Lipschitz-continuous and that the boundedness conditions (4) hold. In addition, we will assume that $\hat{q}+t$ is non-decreasing in time:

$$
\hat{q}\left(\boldsymbol{x}, t_{1}\right)-\hat{q}\left(\boldsymbol{x}, t_{2}\right) \leqslant t_{2}-t_{1} \quad \text { for any } t_{1} \leqslant t_{2} \text { and any } \boldsymbol{x} \in \partial \Omega .
$$

Below we enumerate some properties of the value function that easily follow from the above assumptions.

Proposition 4.1 1. $v(\boldsymbol{x}, t)$ is locally bounded, i.e., for any finite interval $I \subset \mathbb{R}$ there exists a constant $C_{I}$ such that $v(\boldsymbol{x}, t)<C_{I}$ on $\bar{\Omega} \times I$;

2. $v(\boldsymbol{x}, t)$ is Lipschitz-continuous on $\Omega \times \mathbb{R}$;

3. If $t_{2} \geqslant t_{1} \geqslant 0$, then $v\left(\boldsymbol{x}, t_{2}\right) \geqslant v\left(\boldsymbol{x}, t_{1}\right)$ for all $\boldsymbol{x} \in \Omega$.

The standard Optimality Principle for this case [3] is

$$
v(\boldsymbol{x}, t)=\inf _{\boldsymbol{a}(\cdot)} v(\boldsymbol{y}(\Delta t), t+\Delta t) .
$$

The associated Hamilton-Jacobi-Bellman PDE is

$$
\begin{array}{ll}
v_{t}(\boldsymbol{x}, t)+\min _{\boldsymbol{a} \in S_{1}}\left\{\left(\nabla_{x} v(\boldsymbol{x}, t) \cdot \boldsymbol{a}\right) \hat{f}(\boldsymbol{x}, \boldsymbol{a}, t)\right\}=0, & \boldsymbol{x} \in \Omega, t \in \mathbb{R}, \\
v(\boldsymbol{x}, t)=\hat{q}(\boldsymbol{x}, t)+t, & \boldsymbol{x} \in \partial \Omega, t \in \mathbb{R},
\end{array}
$$

and it usually appears as a Mayer problem in the context of finite-horizon optimal control; see, for example, [1] and references therein. By a standard argument, this PDE has a unique viscosity solution on $\bar{\Omega} \times \mathbb{R}$ and that solution is the above-defined value function $v$. The characteristics of the PDE can be again interpreted as optimal trajectories and, if a characteristic emanates from $x_{0} \in \partial \Omega$ at the time $t_{0}$, then $v(\boldsymbol{x}, t)=t_{0}+\hat{q}\left(\boldsymbol{x}_{0}, t_{0}\right)$ for all points $(\boldsymbol{x}, t)$ on that characteristic; see Figure 5 .

In order to compute the value function on $\Omega \times\left[T_{1}, T_{2}\right]$, the PDE has to be solved on $\Omega \times\left[T_{1}, T_{3}\right]$, where $T_{3}>T_{2}$ is sufficiently large to ensure that $v\left(\boldsymbol{x}, T_{2}\right)$ can be computed for all $\boldsymbol{x} \in \Omega$; see Figure 6 The problem is then naturally interpreted as a standard "backward time" finite horizon problem with a terminal value $v\left(\boldsymbol{x}, T_{3}\right)=+\infty$ for all $\boldsymbol{x} \in \Omega$. The resulting Cauchy-Dirichlet boundary value problem can be efficiently solved by higher-order semi-Lagrangian methods (e.g., [13]) or by a variety of Eulerian-framework finite-difference schemes. Such explicit time-marching methods obtain the solution in $O(M N)$ operations, where $M$ is the number of mesh points in the mesh $X$ discretizing $\Omega$ and $N$ is the number of time slices. We also note that an $\Upsilon$-like coefficient is implicitly embedded in this complexity estimate for the finite-difference methods since the CFLtype stability conditions severely limit the "stable" ratio of $N$ to $\sqrt{M}$.

Even though a numerical solution on the entire $\Omega \times\left[T_{1}, T_{2}\right]$ is outside the scope of our paper, we now show how OUMs can efficiently approximate the individual level sets of $v(\boldsymbol{x}, t)$.

First, suppose that we are only interested in an optimal control for a single point $\left(\boldsymbol{x}_{0}, t_{0}\right) \in$ $\Omega \times \mathbb{R}$. Let $\Omega_{1}$ be an open bounded set such that $\bar{\Omega} \subset \Omega_{1}$, and let $\Omega_{2}=\Omega_{1} \backslash\left\{\boldsymbol{x}_{0}\right\}$. We can now

\footnotetext{
3 An obvious exception is the case of a time-discounted control normally used in the infinite horizon problems. There, $\hat{f}(\boldsymbol{y}, \boldsymbol{a}, s)=\hat{f}(\boldsymbol{y}, \boldsymbol{a}) e^{\lambda s}, v(\boldsymbol{x}, t)=e^{-\lambda t} v(\boldsymbol{x}, 0)$, and $\tilde{v}(\boldsymbol{x})$ is therefore quite sufficient.
} 


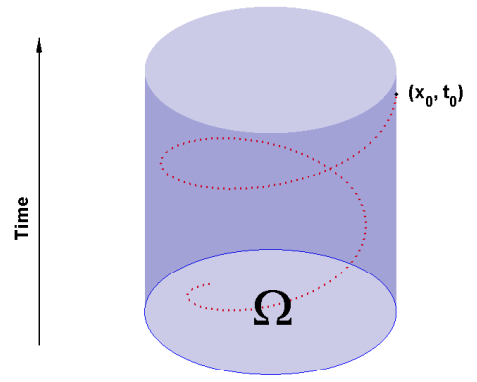

FIG. 5. A spiraling characteristic emanating from $\left(\boldsymbol{x}_{0}, t_{0}\right) \in \partial \Omega \times \mathbb{R}$.

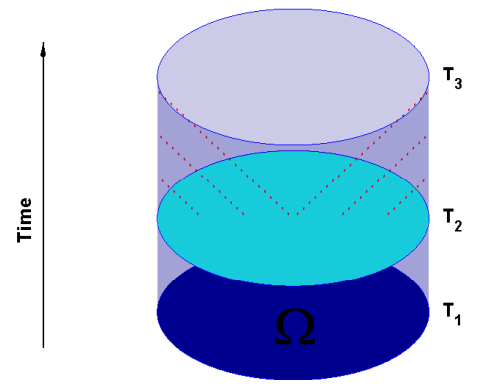

FIG. 6. Solving on $\Omega \times\left[T_{1}, T_{2}\right]$ : the characteristics for all points in $\Omega \times\left\{T_{2}\right\}$ reach the boundary by the time $T_{3}$.

reduce the problem to the static PDE of Section 2 on $\Omega_{2}$ by setting

$$
f(\boldsymbol{y}, \boldsymbol{a}, t)=\hat{f}(\boldsymbol{y}, \boldsymbol{a}, t) ; \quad q\left(\boldsymbol{x}_{0}\right)=t_{0} ; \quad q(\boldsymbol{y})=+\infty \quad \text { for all } \boldsymbol{y} \in \partial \Omega_{1} .
$$

For a trajectory passing through a point $\boldsymbol{x}, \mathcal{J}\left(\boldsymbol{x}, \boldsymbol{a}(\cdot), \boldsymbol{x}_{s}\right)<\infty$ implies $\boldsymbol{x}_{s}=\boldsymbol{x}_{0}$. Thus, for $\boldsymbol{z} \in \Omega_{1}$, $u(\boldsymbol{z})$ is the earliest time when $\boldsymbol{z}$ can be reached from $\boldsymbol{x}_{0}$, and

$$
v\left(\boldsymbol{x}_{0}, t_{0}\right)=\min _{\boldsymbol{z} \in \partial \Omega}\{u(\boldsymbol{z})+\hat{q}(\boldsymbol{z})\} .
$$

If $\boldsymbol{z}$ is a minimizer and $\boldsymbol{y}(t)$ is an optimal trajectory from $\boldsymbol{x}_{0}$ to $\boldsymbol{z}$, then $v(\boldsymbol{y}(t), t)=u(\boldsymbol{z})+\hat{q}(\boldsymbol{z})$ for all $t \in\left[t_{0}, u(\boldsymbol{z})\right]$.

The above procedure effectively yields the characteristic(s) of 32 passing through $\left(\boldsymbol{x}_{0}, t_{0}\right)$ and takes $O(\Upsilon M \log M)$ operations. The resuling optimal trajectory for $\left(\boldsymbol{x}_{0}, t_{0}\right)$ is globally optimalthis is in contrast with "local" methods (e.g., based on Pontryagin's maximum principle) which yield only locally optimal controls and trajectories. Nevertheless, since the computational cost of the latter methods is independent of the dimension of state-space, the described procedure would not be worthwhile just to recover those characteristic(s) of (32) that pass through a single point in $\Omega \times \mathbb{R}$. Fortunately, a similar construction can be used to recover all characteristics lying in the same level set of $v(\boldsymbol{x}, t)$ without increasing the computational cost 4

\footnotetext{
${ }^{4}$ As pointed out by Sethian and Shan [28], if the time is reversed, the time-independent min-time-from-boundary value function of Section 2 can be interpreted as an answer to a different question: What is the latest time when one can depart from each $\boldsymbol{x} \in \Omega$ and still leave $\bar{\Omega}$ before the specified time $T$ ? Our discussion here is somewhat more general since in [28] the setting is limited to isotropic time-optimal control problems with zero exit/entrance time-penalties.
} 


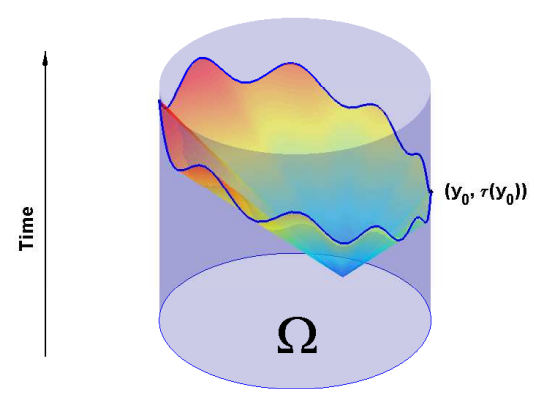

FIG. 7. A level set of $v(\boldsymbol{x}, t)$ and its intersection with $\partial \Omega \times \mathbb{R}$.

OBSERVATION 4.2 Since $v(\boldsymbol{x}, t)$ is constant along the characteristics, two characteristics emanating from the points $\left(\boldsymbol{x}_{0}, t_{0}\right)$ and $\left(\boldsymbol{x}_{1}, t_{1}\right)$ on $\partial \Omega \times \mathbb{R}$ can collide only if $t_{0}+\hat{q}\left(\boldsymbol{x}_{0}, t_{0}\right)=$ $t_{1}+\hat{q}\left(\boldsymbol{x}_{1}, t_{1}\right)$. The assumption on monotonicity of $\hat{q}+t$ implies that every level set of $v(\boldsymbol{x}, t)$ in $\bar{\Omega} \times \mathbb{R}$ is a graph of some function over $\bar{\Omega}$ and transversally intersects the boundary $\partial \Omega \times \mathbb{R}$; see Figure 7 We emphasize that this observation is valid even when the speed $\hat{f}$ is not a monotone function of time. [Moreover, even if $F_{1}=0$ and the reachable set is not the entire $\Omega$, each level set of $v$ will be a (possibly infinite) surface emanating from a single curve of boundary conditions on $\partial \Omega \times \mathbb{R}$.]

We now use OUMs to reconstruct the level set $v(\boldsymbol{x}, t)=T$ in $\bar{\Omega} \times \mathbb{R}$ by solving a min-time-fromboundary problem on $\Omega$. For every $\boldsymbol{y}_{0} \in \partial \Omega$, let $\tau\left(\boldsymbol{y}_{0}\right)$ be the time such that $\tau\left(\boldsymbol{y}_{0}\right)+\hat{q}\left(\boldsymbol{y}_{0}, \tau\left(\boldsymbol{y}_{0}\right)\right)=$ $T$ and let

$$
\begin{aligned}
f(\boldsymbol{y}, \boldsymbol{a}, t) & =\hat{f}(\boldsymbol{y},-\boldsymbol{a}, T-t) & & \text { for all } \boldsymbol{y} \in \overline{\boldsymbol{\Omega}}, \boldsymbol{a} \in S_{1}, t \in \mathbb{R} ; \\
q\left(\boldsymbol{y}_{0}\right) & =\hat{q}\left(\boldsymbol{y}_{0}, \tau\left(\boldsymbol{y}_{0}\right)\right) & & \text { for all } \boldsymbol{y}_{0} \in \partial \Omega .
\end{aligned}
$$

THEOREM 4.3 Let $u(x)$ be the value function of the min-time-from-boundary problem specified above. Then

$$
u(x)=t \quad \Leftrightarrow \quad v(\boldsymbol{x}, T-t)=T .
$$

Proof. Consider a particular control $\boldsymbol{a}(\cdot)$ and a starting point $\boldsymbol{y}_{0} \in \partial \Omega$ such that the corresponding trajectory $\boldsymbol{y}(\cdot)$ passes through $\boldsymbol{x}$. Let $t_{a}=\mathcal{J}\left(\boldsymbol{x}, \boldsymbol{a}(\cdot), \boldsymbol{y}_{0}\right)$ and define

$$
\begin{aligned}
\hat{\boldsymbol{y}}^{\prime}(s) & =\hat{f}(\hat{\boldsymbol{y}}(s), \hat{\boldsymbol{a}}(s), s) \hat{\boldsymbol{a}}(s), \\
\hat{\boldsymbol{y}}\left(T-t_{a}\right) & =\boldsymbol{x},
\end{aligned}
$$

where $\hat{\boldsymbol{a}}(s)=-\boldsymbol{a}(T-s)$ and

$$
T-t_{a} \leqslant s \leqslant T-\hat{q}\left(\boldsymbol{y}_{0}, \tau\left(\boldsymbol{y}_{0}\right)\right)=\tau\left(\boldsymbol{y}_{0}\right) .
$$

Then, by Gronwall's lemma, $\hat{\boldsymbol{y}}(s)=\boldsymbol{y}(T-s)$ and

$$
\widehat{\mathcal{J}}\left(\boldsymbol{x}, \hat{\boldsymbol{a}}(\cdot), T-t_{a}\right)=\tau\left(\boldsymbol{y}_{0}\right)+\hat{q}\left(\boldsymbol{y}_{0}, \tau\left(\boldsymbol{y}_{0}\right)\right)=T .
$$

Thus, $v\left(\boldsymbol{x}, T-t_{a}\right) \leqslant T$ for $t_{a}$ arbitrarily close to $u(\boldsymbol{x})$, yielding $v(\boldsymbol{x}, T-u(\boldsymbol{x})) \leqslant T$.

Let $t_{1}$ be such that $v\left(\boldsymbol{x}, t_{1}\right)=T$. (Note that $t_{1} \geqslant T-u(\boldsymbol{x})$ by the monotonicity of $v$.) If $\hat{\boldsymbol{a}}(s)$ is an optimal control, then

$$
v\left(\boldsymbol{x}, t_{1}\right)=\widehat{\mathcal{J}}\left(\boldsymbol{x}, \hat{\boldsymbol{a}}(\cdot), t_{1}\right)=T .
$$


Thus, if $\boldsymbol{y}_{0}=\hat{\boldsymbol{y}}\left(T\left(\boldsymbol{x}, \hat{\boldsymbol{a}}(\cdot), t_{1}\right)\right)$, then $\tau\left(\boldsymbol{y}_{0}\right)+\hat{q}\left(\boldsymbol{y}_{0}, \tau\left(\boldsymbol{y}_{0}\right)\right)=T$ and $\boldsymbol{a}(s)=-\hat{\boldsymbol{a}}(T-s)$ is a valid control for $u(x)$. Hence,

$$
u(\boldsymbol{x}) \leqslant \mathcal{J}\left(\boldsymbol{x}, \boldsymbol{a}(\cdot), \boldsymbol{y}_{0}\right)=T-t_{1} .
$$

Therefore, $t_{1}=T-u(\boldsymbol{x})$, which completes the proof.

REMARK 4.4 The above proof relies on the existence of an optimal control for $v\left(\boldsymbol{x}, t_{1}\right)$. A more general proof can be constructed: an $\epsilon$-suboptimal trajectory $\hat{\boldsymbol{y}}(\cdot)$ for $v\left(\boldsymbol{x}, t_{1}\right)$ will reach some $\boldsymbol{y}_{0} \in$ $\partial \Omega$ at the time $\tau_{1}$ such that

$$
\tau\left(\boldsymbol{y}_{0}\right)+\hat{q}\left(\boldsymbol{y}_{0}, \tau\left(\boldsymbol{y}_{0}\right)\right) \leqslant \tau_{1}+\hat{q}\left(\boldsymbol{y}_{0}, \tau_{1}\right) \leqslant \tau\left(\boldsymbol{y}_{0}\right)+\hat{q}\left(\boldsymbol{y}_{0}, \tau\left(\boldsymbol{y}_{0}\right)\right)+\epsilon .
$$

Reparameterizing this trajectory backwards in time and starting along it at the time $\hat{q}\left(\boldsymbol{y}_{0}, \tau\left(\boldsymbol{y}_{0}\right)\right)$, we obtain for $u(x)$ a valid trajectory whose cost is close to $T-\widehat{\mathcal{J}}\left(\boldsymbol{x}, \hat{\boldsymbol{a}}(\cdot), t_{1}\right)$ by the Lipschitzcontinuity of $\hat{f}$ and $\hat{q}$.

We note that OUM used on the above min-time-from-boundary problem yields all the characteristics of (32) inside the level set $v(\boldsymbol{x}, t)=T$ (and thus the optimal trajectories for all the points in that level set) in $O(\Upsilon M \log M)$ operations. In addition, we list two special problems for which our approach should be particularly useful:

1. Suppose that an additional/independent starting cost $K(\boldsymbol{x}, t)$ is incurred and the goal is to choose the starting position/time $(\boldsymbol{x}, t)$ to minimize $K$ while ensuring that we can exit $\Omega$ before some fixed time $T$ (i.e., $v(\boldsymbol{x}, t) \leqslant T$ should be satisfied). If $u(\boldsymbol{x})$ is defined as in Theorem 4.3 , then $K$ has to be minimized over the set $\{(\boldsymbol{x}, t) \mid \boldsymbol{x} \in \Omega, 0 \leqslant t \leqslant T-u(\boldsymbol{x})\}$.

2. More generally, it is often necessary to find a control/trajectory satisfying multiple integral constraints (a recent discussion of this problem can be found in [22]). In the non-autonomous case, if one of the constraints is based on the time-to-exit, the above techniques will allow one to restrict the computational domain to a smaller subset of $\Omega \times I$ before checking whether other constraints can be satisfied.

We also hope that similar "reduction-to-static-case" methods might be applicable for more general time-dependent problems.

\section{Conclusions}

We have analyzed a min-time-from-boundary non-autonomous control problem and interpreted its value function as the unique viscosity solution of the Dirichlet problem for a static HamiltonJacobi-Bellman PDE. We have described a non-iterative semi-Lagrangian Ordered Upwind Method to obtain a first-order numerical approximation on the mesh $X$ in $O(M \log M)$ operations, where $M$ is the number of mesh points. We have illustrated our method with several non-autonomous time-optimal control problems.

We note that our approach is, in some sense, the opposite of a common prior technique, which converted a Dirichlet problem for $u(x)$ into a Cauchy problem for a function $\psi(\boldsymbol{x}, t)$ with the property that

$$
u(\boldsymbol{x})=t \Leftrightarrow \psi(\boldsymbol{x}, t)=0
$$

(see, for example, [23] or [27] and references therein). Supposing $u=q$ is constant on the boundary, $\psi(\boldsymbol{x}, 0)$ was initialized as a signed distance to $\partial \Omega$ on some bigger domain $\Omega_{1}$ and the level set 
methods [24] were used to evolve $\psi$ forward in time. We believe that the original motivation for such an approach was the absence of computationally efficient methods for non-linear hyperbolic boundary value problems. In this paper, we have presented an alternative-a fast non-iterative solver-for the case when the Hamiltonian $H$ is convex and homogeneous of degree one in the first argument. The advantage, of course, is the reduced dimensionality of the computational domain and the overall reduction of the time required to obtain a solution on a given grid. Moreover, in Section 4 we have demonstrated that fast boundary value solvers can also be used to efficiently obtain individual level sets of the viscosity solution to a particular time-dependent HJB PDE.

We believe that the general idea of space-marching is applicable to a much wider class of PDEs. We note that OUMs were already used to solve quasi-variational inequalities in the context of hybrid control [31], a linear Liouville PDE arising in the context of seismic imaging [16], and, more recently, a special class of quasi-linear PDEs arising in invariant manifold approximations [15].

The presented idea of a purely static reformulation is largely independent of the simplifying assumptions (e.g., $F_{1}>0$ ) used here to obtain a simple and efficient numerical method. Extending OUMs to handle the general non-small-time-controllable dynamics and non-holonomic constraints will make the method applicable to a wide range of realistic problems in optimal control and robotic navigation. Other topics of current research include extensions of OUMs to non-convex Hamiltonians and design of provably higher-order non-iterative methods.

\section{Acknowledgments}

The author thanks J. A. Sethian, Y. Shan, L. C. Evans, and P. E. Souganidis for motivating discussions and helpful suggestions. The first draft of this paper was completed during a visit to Lawrence Berkeley National Laboratory, and the author is grateful to LBNL for its hospitality.

The author's research is supported by the National Science Foundation grants DMS-0514487 and CTS-0426787.

\section{REFERENCES}

1. Bardi, M., \& CApuzzo Dolcetta, I. Optimal Control and Viscosity Solutions of Hamilton-JacobiBellman Equations. Birkhäuser Boston (1997). Zbl 0890.49011 MR 1484411

2. BARDi, M., \& FALCONE, M. An approximation scheme for the minimum time function. SIAM J. Control Optim. 28 (1990), 950-965. Zbl 0723.49024 MR 1051632

3. Bellman, R. Dynamic Programming. Princeton Univ. Press, Princeton NJ (1957).

4. BouÉ, M., \& DupuIs, P. Markov chain approximations for deterministic control problems with affine dynamics and quadratic cost in the control. SIAM J. Numer. Anal. 36 (1999), 667-695. Zbl 0933.65073 MR 1681057

5. Broucke, M., Di Benedetto, M. D., Di Gennaro, S., \& Sangiovanni-Vincentelli, A. L. Theory of optimal control using bisimulations. In Hybrid Systems: Computation and Control (HSCC2000), Lecture Notes in Comput. Sci. 1790, Springer (2000), 89-102. Zbl 0963.93059

6. Broucke, M., Di Benedetto, M. D., Di Gennaro, S., \& Sangiovanni-Vincentelli, A. L. Optimal control using bisimulations: implementation. In Hybrid Systems: Computation and Control (HSCC2001), Lecture Notes in Comput. Sci. 2034, Springer (2001), 175-188. Zbl 0995.49021

7. Crandall, M. G., Evans, L. C., \& Lions, P-L. Some properties of viscosity solutions of HamiltonJacobi equations. Trans. Amer. Math. Soc. 282 (1984), 487-502. Zbl 0543.35011 MR 0732102 
8. Crandall, M. G., \& Lions, P-L. Viscosity solutions of Hamilton-Jacobi equations. Trans. Amer. Math. Soc. 277 (1983), 1-42. Zbl 0599.35024 MR 0690039

9. DANIElsson, P.-E. Euclidean distance mapping. Computer Graphics Image Processing 14 (1980), 227248.

10. Evans, L. C., \& Souganidis, P. E. Differential games and representation formulas for solutions of Hamilton-Jacobi-Isaacs equations. Indiana Univ. Math. J. 33 (1984), 773-797. MR 0756158

11. FAlcone, M. The minimum time problem and its applications to front propagation. Motion by Mean Curvature and Related Topics (Trento, 1992), de Gruyter, New York (1994), 70-88. Zbl 0803.65080 MR 1277392

12. FAlCONE, M. A numerical approach to the infinite horizon problem of deterministic control theory. Appl. Math. Optim. 15 (1987), 1-13; Corrigenda, 23 (1991), 213-214. Zbl 0715.49025 MR 1086470

13. Falcone, M., \& Ferretti, R. Discrete time high-order schemes for viscosity solutions of HamiltonJacobi-Bellman equations. Numer. Math. 67 (1994), 315-344. Z Zbl 0791.65046 MR 1269500

14. Gonzales, R., \& Rofman, E. On deterministic control problems: an approximate procedure for the optimal cost, I, the stationary problem. SIAM J. Control Optim. 23 (1985), 242-266.

15. Guckenheimer, J., \& Vladimirsky, A. A fast method for approximating invariant manifolds. SIAM J. Appl. Dynam. Systems 3 (2004), 232-260. Zbl 1059.37019|| MR 2114735

16. Fomel, S., \& Sethian, J. A. Fast-phase space computation of multiple arrivals. Proc. Nat. Acad. Sci. USA 99 (2002), 7329-7334. Zbl 1002.65113 MR 1907838

17. Junge, O., \& OsIngA, H. M. A set oriented approach to global optimal control. ESAIM: Control Optim. Calc. Var. 10 (2004), 259-270. Zbl 1072.49014 MR 2083487

18. KAO, C. Y., Osher, S., \& QIAN, J. Lax-Friedrichs sweeping scheme for static Hamilton-Jacobi equations. J. Comput. Phys. 196 (2004), 367-391. Zbl 1053.65088 MR 2054347

19. KRUZhKov, S. N. Generalized solutions of the Hamilton-Jacobi equations of the eikonal type. Math. USSR-Sb. 27 (1975), 406-445. Zbl 0369.35012

20. Kushner, H. J. Probability Methods for Approximations in Stochastic Control and for Elliptic Equations. Springer, New York (1977). Zbl 0547.93076 MR 0469468

21. Kushner, H. J., \& Dupuis, P. G. Numerical Methods for Stochastic Control Problems in Continuous Time. Academic Press, New York (1992). Zbl 0754.65068 MR 1217486

22. Mitchell, I., \& SASTRY, S. Continuous path planning with multiple constraints. UC Berkeley Engineering Research Laboratory Technical Report (UCB/ERL M03/34) (2003).

23. OSHER, S. A level set formulation for the solution of the Dirichlet problem for Hamilton-Jacobi equations. SIAM J. Math. Anal. 24 (1993), 1145-1152. Zbl 0804.35021 MR 1234009

24. OShER, S., \& SETHiAn, J. A. Fronts propagating with curvature-dependent speed: algorithms based on Hamilton-Jacobi formulations. J. Comput. Phys. 79 (1988), 12-49. Z Zbl 0659.65132 MR 0965860

25. Qian, J., \& S YMES, W. W. Paraxial eikonal solvers for anisotropic quasi-P traveltimes. J. Comput. Phys. 173 (200), 256-2781. Zbl 1056.35042

26. QIAN, J., \& SYMES, W. W. A paraxial formulation for the viscosity solution of quasi-P eikonal equations. Comput. Math. Appl. 46 (2003), 1691-1701. Zbl 1049.35071 MR 2024241

27. Sethinan, J. A. Level Set Methods and Fast Marching Methods: Evolving Interfaces in Computational Geometry, Fluid Mechanics, Computer Vision, and Materials Science. Cambridge Univ. Press (1999). Zbl 0973.76003 MR 1700751

28. Sethian, J. A., \& Shan, Y. Extensions of fast marching methods to time-dependent cost functions. In progress.

29. Sethian, J. A., \& Vladimirsky, A. Ordered upwind methods for static Hamilton-Jacobi equations. Proc. Nat. Acad. Sci. USA 98 (2001), 11069-11074. Zbl 1002.65112 MR 1854545 
30. Sethian, J. A., \& Vladimirsky, A. Ordered upwind methods for static Hamilton-Jacobi equations: theory and algorithms. SIAM J. Numer. Anal. 41 (2003), 325-363. Zbl 1040.65088 MR 1974505

31. Sethian, J. A., \& Vladimirsky, A. Ordered upwind methods for hybrid control. Hybrid Systems: Computation and Control (HSCC 2002), Lecture Notes in Comput. Sci. 2289, Springer (2002), 393-406. Zbl 1050.93042

32. Soner, M. H. Optimal control problems with state-space constraint. SIAM J. Control Optim. 24 (1986), 552-561 and 1110-1122. Zbl 0619.49013 MR 0861089

33. Soravia, P. Generalized motion of a front propagating along its normal direction: a differential games approach. Nonlinear Anal. 22 (1994), 1247-1262. Zbl 0814.35140 MR 1279982

34. Tsai, Y.-H. R., Cheng, L.-T., Osher, S., \& ZHAO, H.-K. Fast sweeping algorithms for a class of Hamilton-Jacobi equations. SIAM J. Numer. Anal. 41 (2003), 673-694. Zbl 1049.35020 MR 2004194

35. VLADIMIRSKY, A. Non-iterative methods for boundary value problems. In progress.

36. ZhaO, H.-K. Fast sweeping method for eikonal equations. Math. Comp. 74 (2005), 603-627. Zbl 1070.65113 MR 2114640 